\section{Adriano Chiò}

\author{
A. Chiò (ه) \\ Department of Neuroscience, \\ University of Turin, \\ Via Cherasco 15 \\ 10126 Torino, Italy \\ e-mail: schiffer@golgi.molinette.unito.it
}

\section{ISIS Survey: an international study on the diagnostic process and its implications in amyotrophic lateral sclerosis}

\begin{abstract}
bar level and when fasciculations were present. The major causes of diagnostic delay were unfamiliarity of the physician with the disease, unusual clinical presentation, coexistence of other diseases to which clinical symptomatology was attributed, misleading findings or misinterpretation of neuro-radiological or neurophysiological findings. The diagnostic delay has several implications for patients and their families, including mismanagement, delay in establishing appropriate and individualized pharmacological and symptomatic therapies, and difficulty in planning personal and familiar future.
\end{abstract}

Key words Amyotrophic lateral sclerosis $\cdot$ Diagnosis $\cdot$ Misdiagnosis

\section{Introduction}

Amyotrophic lateral sclerosis (ALS) is a disease of adult life. It is characterized by the involvement of spinal motor neurons, resulting in muscle weakness and wasting of bulbar motor neurons (with dysarthria, dysphagia and tongue wasting) and of pyramidal tracts (with increased tendon reflexes and spasticity) $[18,22]$. ALS often begins insidiously with a slight weakness of hand or feet muscles and fasciculations, sometimes associated with cramps, with a subsequent appearance of mild leg spasticity. Often, ALS may be difficult to distinguish from other diseases until a full clinical picture is present. Some previous studies reported that in a number of patients there is a relatively long delay before the diagnosis is made, and that some patients are initially misdiagnosed, with consequent inap- propriate or even harmful management [2, 3, 20, 16]. Therefore, the ISIS Survey was conducted to identify the pathway followed by ALS patients from the first symptoms to the confirmation of diagnosis, in order to determine which factors delay the diagnostic process and how they can be modified to limit delays in accurate diagnosis and subsequent treatment.

\section{Materials and methods}

The study was performed in 6 countries (Argentina, Brazil, Germany, Italy, Spain and USA). A total of 201 patients were included (30 from each country except Italy $=31$, and USA $=50$ ). Only patients with an ALS diagnosis confirmed within 3 years were considered. ALS diagnosis was based on World Federation of Neurology (WFN) criteria for defined and probable ALS [5]. The patients were recruited randomly through lists provided by the physician or 
national ALS associations. Stratification for age, sex, and initial symptoms (bulbar versus spinal) was requested.

A common structured case report was used for all countries, which included information about patient's demographic characteristics and clinical history with particular attention to the various steps taken towards the final diagnosis of ALS (consultations with physicians, examinations performed, key events in social life, use of assistive devices). When considered necessary, more information was requested from the physicians. All patients gave written informed consent prior to participation in the study.

\section{Results}

A total of 201 patients were recruited, $118(58.7 \%)$ men and $83(41.3 \%)$ women, with a mean age of 56.1 years. Initial symptoms (Table 1) were at bulbar level in 37 $(18.4 \%)$, and at spinal level in $164(81.6 \%)$. No differences in demographic characteristics were observed between various countries, other than there was a greater proportion of women in the Brazilian cohort. After the onset of symptoms, the first physician seen by most patients $(60 \%)$ was the general practitioner (GP), followed by the traumatologist/orthopedist (15\%) and then the neurologist $(11 \%)$. The median time between onset of symptoms and first consultation with a physician (excluding 7 patients) was 5.6 months (range; Spain $=2$ months, Germany = 8 months). In $63 \%$ of cases, the patient was referred to a neurologist after a first consultation with the GP. For the remaining $37 \%$, referral after the first consultation was to an orthopedist, a rheumatologist, or an otolaryngologist. The first specialist seen was usually a neurologist (48\%; Italy $=70 \%$ ), followed by a traumatologist/orthopedic surgeon $(35 \%$; Spain $=56 \%$, and Argentina $=50 \%)$, and by an otolaryngologist $(4.5 \%)$. The mean time between the first visit and the first consultation with a neurologist was 6 months (range; Germany $=3$ months to Spain $=9$ months). Finally, the mean time needed for a neurologist to reach the diagnosis of ALS was 7 months (range; Ger-

Table 1 Symptoms appearing during the first 3 months (as indicated by the patients) ${ }^{\mathrm{a}}$

\begin{tabular}{lrr}
\hline Symptoms & $n$ & $\%$ \\
\hline Motor disorders (spinal level): & & \\
$\quad$ Upper limbs & 69 & 34.3 \\
Lower limbs & 71 & 35.3 \\
Both & 13 & 6.5 \\
Bulbar disorders & 37 & 18.4 \\
Asthenia & 20 & 10.0 \\
Muscular cramps & 48 & 23.9 \\
Stiffness & 5 & 2.5 \\
Amyotrophy & 17 & 8.5 \\
Fasciculations & 38 & 18.9 \\
Dyspnoea & 7 & 3.5 \\
Balance disorders & 5 & 2.5 \\
\hline
\end{tabular}

a The total exceeds $100 \%$ because several patients indicated more than one symptom
Table 2 Examinations performed before diagnosis, by country (percent of cases)

\begin{tabular}{lrlllll}
\hline Country & EMG & MRI & CT & CSF & MEP & Biopsy \\
\hline Argentina & 100 & 78 & 37 & 13 & 33 & 10 \\
Brazil & 98 & 53 & 43 & 43 & 3 & 7 \\
Germany & 97 & 70 & 67 & 73 & 73 & 50 \\
Italy & 100 & 90 & 61 & 29 & 55 & 6 \\
Spain & 93 & 70 & 43 & 13 & 20 & 3 \\
USA & 94 & 84 & 46 & 16 & 22 & 14 \\
\hline
\end{tabular}

EMG, electromyography and electroneurography; MRI, magnetic resonance imaging of head and neck; CT, computed tomography of head and neck; CSF, cerebrospinal fluid examination; MEP, motor evoked potentials; Biopsy, muscle and/or nerve biopsy

Table 3 Most common misdiagnoses

\begin{tabular}{lr}
\hline Diagnoses & $\%$ \\
\hline $\begin{array}{lr}\text { Discal hernia/medullar } \\
\quad \text { compression }\end{array}$ & 12 \\
Arthrosis/periarthitis & 9 \\
Narrow medullar canal & 4 \\
Osteoporosis & 2 \\
Laryngitis/chronic tonsillitis & 2 \\
Cerebrovascular accident & 3 \\
Thyroid dysfunction & 1 \\
Parkinson's disease & 1 \\
Multiple sclerosis & 1 \\
Others & 10 \\
\hline Overall & 45 \\
\hline
\end{tabular}

many $=5$ months, Spain $=5$ months to Italy (excluding one patient $)=10$ months). When a neurologist was the first or the second physician seen by the patients the mean time to reach the diagnosis of ALS was shorter (17 months when first seen by neurologist; 17 months when seen by neurologist second; 19 months when seen by neurologist third; 21 months when seen by neurologist fourth or fifth).

The mean time to diagnosis was related to the site of first symptoms (bulbar, 14 months; upper limbs, 15 months; lower limbs, 21 months), and to the presence of fasciculations (presence of fasciculations, 15 months; absence of fasciculations, 19 months). Laboratory examinations performed before the diagnosis were similar in all countries (Table 2). In particular, electromyography (EMG) was performed in almost all patients, and magnetic resonance imaging (MRI) and/or computed tomography $(\mathrm{CT})$ of head and neck in most. A different attitude towards more invasive procedures such as cerebrospinal fluid examination and nerve or muscular biopsy was identified. Such procedures were performed in few patients in most countries, except in Germany. Misdiagnoses were relatively frequent, occurring in about $45 \%$ of patients (Table 3). These were made by neurologists (28\%), GPs (29\%) and traumatologists/orthopedic surgeons (26\%). 


\section{Discussion}

In an era of increasing reliance on sophisticated brain imaging, electrophysiological and molecular testing, the diagnosis of ALS is still based on clinical features, only supplemented by laboratory findings. The diagnostic criteria for ALS proposed by the WFN reflect this observation, giving more weight to clinical aspects and relegating laboratory tests to an exclusion or, at best, to a supporting role in the diagnostic process [3]. However, classically the diagnosis of ALS has been considered straightforward [18]. Yet according to several authors, the mean time delay between onset of symptoms and established diagnosis of ALS is over 12 months [7, 15], consistent with this study, and $26-42 \%$ of patients are initially misdiagnosed $[2,3,20,16]$. This delay in diagnosis is dramatically long when considering that the median survival of ALS patients is between 30-36 months [7].

The time delay between the onset of symptoms and the final diagnosis of ALS has been found to be related both to some characteristics inherent to the clinical presentation and to the time needed for a patient to be referred to a neurologist. The presence of some signs (dysarthria, dysphagia and fasciculations) appears to shorten the diagnostic process. This observation is consistent with a previous study conducted in China, England and Germany to investigate the attitude of neurologists toward ALS diagnosis [13]. It was observed that bulbar involvement and presence of fasciculation were considered the most relevant signs for establishing ALS diagnosis.

Taking into consideration the findings of this and other studies $[2,3,20,16]$, four possible causes of delay in diagnosis and misdiagnosis of ALS may be identified:

- Coexistence of other diseases, to which the clinical symptomatology is attributed.

- Misleading findings, or misinterpretation, of neuro-radiological and neuro-physiological examinations. It is known that in a number of patients with ALS, MRI or CT scan of the spinal column can show pathological findings possibly indicative of disease other than ALS, especially of cervical spondylopathic myelopathy. In these instances, it is quite difficult to establish if the abnormalities noted on MRI or CT scan are really sufficient to cause the observed clinical picture [22]. Interpretation of EMG findings may also create some uncertainty in diagnosing patients with suspected ALS. The most widely used criteria for the electrophysiological diagnosis of ALS are those proposed by Lambert [12]. However, some patients with ALS fail to fulfill Lambert's criteria, especially those with minimal or absent spinal involvement. For example, Behnia and Kelly [1] found that 38\% of 133 ALS patients did not correspond to Lambert's criteria - due to the presence of abnormal nerve conduction, attributable to a mild axonal polyneuropathy, or the absence of widespread muscle denervation.
Table 4 Implications of diagnostic delay and misdiagnosis for ALS patients

1) Mismanagement

2) Psychological impact to the patients and their families

3) Delay in establishing and organizing appropriate and individualized pharmacological and support therapy

4) Obstacles in including the patient in therapeutic trials

5) Delay in obtaining financial support and delivering equipment and supplies by government agencies or third party payers

6) Difficulty in planning personal and familial future

- Unfamiliarity with the disease by the GP or the specialist (often not a neurologist) who first sees the patient. This is demonstrated by the longer diagnostic delay observed in patients seen later on by a neurologist in the diagnostic pathway. In almost all cases in this study, a neurologist performed ALS diagnosis, even if another physician primarily saw the patient; this observation probably reflects the difficulty for a non-neurologist to establish that the clinical presentation is due to a neurological disorder.

- Unusual presentation of ALS, such as hemiparetic (Mills' variant) [16], respiratory [11], or pseudopolyneuritic onset [20].

The implications of late diagnosis and misdiagnosis for the patient, their families and the physician can be immense (Table 4). The major problem due to misdiagnosis is probably mismanagement. Before the diagnosis of ALS, many patients may undergo unnecessary, costly and sometimes painful examinations, and receive inadequate or even dangerous treatments, for example, spinal surgery. In addition, some 'supposedly' appropriate treatments can be inadequately performed in ALS patients, such as physical therapy $[10,14,19]$.

The psychological impact of misdiagnosis should not be overlooked. Often the patients are erroneously diagnosed to have a mild, reversible disease. They may, therefore, develop expectations of recovering or a slow progressive impairment of functions. When they compare these expectations to the relentlessly progressive course of ALS, and are told that the disease is, in fact, untreatable, they can develop a depressive reaction, sometimes associated with a refusal of further therapies and a loss of trust toward the physician.

Recently, glutamate antagonist riluzole has offered fresh hope in reaching the goal of developing an effective therapy for ALS [4]. The emergence of new ALS therapies further emphasizes the need for early diagnosis, as pharmacological therapies could be more effective when administered in the early stages of the disease $[8,17]$.

In addition to pharmacological intervention, patients can be supported in several ways, such as with symptomatic treatments (for example, drugs useful in relieving spastic symptoms, controlling drooling, etc), physical therapy, and assistive devices [21]. As these supportive 
measures are tailored to the specific needs of each patient, subsequent delay in diagnosis is likely to affect their efficacy. Moreover, a patient diagnosed in a relatively advanced stage has lost the opportunity to enroll in therapeutic trials, since these usually only recruit patients with a relatively mild disability. The patients are thus prevented from obtaining the possible therapeutic and psychological benefits of experimental therapies $[2,9]$, potentially causing further frustration due to feelings of hopelessness [6].

The excessive delay in reaching the correct diagnosis also has implications on delivering supportive treatments, equipment and supplies. Generally, the possibility of access to special social services from local communities, obtaining equipment and supplies from governmental agencies or third party payers and receiving disability benefits from the social security system is related to the recognition of a permanent disability, and therefore to a correct diagnosis of ALS.

Finally, the establishment of an early diagnosis of ALS gives the patient the chance to plan their future. In the early stages of the disease, patients have to think about their ability to work and plan for their financial future. Moreover, they need to make decisions about more invasive support therapies which will be necessary later in the course of the disease (i.e., feeding by nasogastric tube or percutaneous gastrostomy; protection of the airway with tracheostomy or full-time mechanical non-invasive ventilation) $[9,23]$.

In conclusion, delay of diagnosis and misdiagnosis of ALS is very frequent, even in countries with different health care systems. This inevitably has considerable neg- ative medical, financial, and psychological impacts on the patient. Despite this, 'delay' of diagnosis in some cases is probably unavoidable, as the neurologist often needs necessary follow-up time to determine with confidence whether or not ALS is present. However, in the majority of instances it is unnecessary and unjustified, since the differentiation of ALS from other diseases is not usually difficult when support examinations are correctly interpreted in the light of the symptomatology. Such symptoms include the presence of widespread muscular weakness and atrophy, pyramidal signs in already denervated muscles, dysarthria and swallowing difficulties (associated with the absence of sensory symptoms, pain, extraocular movement impairment, and sphincter abnormalities). However, a high level of suspicion, especially in cases with unusual presentation, is necessary. The provisions for education of non-neurologists, both GPs and other specialists, should be undertaken, in order to facilitate the prompt referral to a neurologist of all patients with a clinical picture resembling ALS. A correct and early diagnosis of ALS may help the physician to spare the patient from prolonged hospitalization, and expensive, sometimes painful, diagnostic procedures and treatments. Moreover, a correctly diagnosed patient in the early stages of the disease may have access to comprehensive care, including psychological support, directed to relieve their major disability and sufferings.

Acknowledgements The present study was supported by an unrestricted grant by Rhône-Poulenc Rorer, Antoine, France. Patients and physicians participating in the study are gratefully acknowledged.

\section{References}

1. Behnia M, Kelly JJ (1991) Role of electromyography in amyotrophic lateral sclerosis. Muscle Nerve 14:12361241

2. Belsh JM, Schiffman PL (1990) Misdiagnosis in patients with amyotrophic lateral sclerosis. Arch Intern Med 150: 2301-2305

3. Belsh JM, Schiffman PL (1996) The amyotrophic lateral sclerosis (ALS) patient perspective on misdiagnosis and its repercussions. J Neurol Sci 139 [Suppl] : 110-116

4. Bensimon G, Lacomblez L, Meininger $\mathrm{V}$, for the ALS/Riluzole Study Group (1994) A controlled trial of riluzole in amyotrophic lateral sclerosis. N Eng J Med 330:585-591
5. Brooks BR and Subcommittee on Motor Neuron Diseases/Amyotrophic Lateral Sclerosis of the World Federation of Neurology Research Group on Neuromuscular Diseases and the El Escorial 'Clinical limits of amyotrophic lateral sclerosis' workshop contributors (1994) El Escorial World Federation of Neurology criteria for the diagnosis of amyotrophic lateral sclerosis. J Neurol Sci 124 [Suppl]: 98-107

6. Brooks BR, Sufit RL, DePaul R, et al (1991) Design of clinical therapeutic trials in amyotrophic lateral sclerosis. Adv Neurol 56:521-546

7. Chancellor AM, Slattery JM, Fraser H, et al (1993) The prognosis of adult-onset motor neuron disease: a prospective study based on the Scottish Motor Neuron Disease Register. J Neurol 240:339-346

8. Eisen A (1996) Therapeutic opportunities in amyotrophic lateral sclerosis. The Neurologist 2:85-95
9. Gelinas D (1997) Patient and caregiver communications and decisions. Neurology [Suppl 4]: S 9-S 14

10. Goldblatt D (1992) Caring for patients with amyotrophic lateral sclerosis. In: Smith RA (ed) Handbook of amyotrophic lateral sclerosis, New York: Marcel Dekker Inc, pp 271-288

11. Kuisma MJ, Saarinen KV, Teirmaa HT (1993) Undiagnosed amyotrophic lateral sclerosis and respiratory failure. Acta Anaesthesiol Scand 37:628-630

12. Lambert EH (1969) Electromyography in amyotrophic lateral sclerosis. In: Norris FH Jr, Kurland LT (eds) Motor Neuron Disease. Grune \& Stratton, New York, pp 135-153

13. Li T-M, Swash M, Alberman E, et al (1991) Diagnosis of motor neuron disease by neurologists: a study in three countries. J Neurol Neurosurg Psychiatry 54:980-983 
14. Mitsumoto H, Chad DA, Pioro EP (1998) Physical rehabilitation. In: Mitsumoto H, Chad DA, Pioro EP (eds) Amyotrophic lateral sclerosis. FA Davis Company, Philadelphia, pp 360-381

15. Norris FH, Shepherd R, Denys E, et al (1993) Onset, natural history and outcome in idiopathic adult motor neuron disease. J Neurol Sci 118:48-55

16. O'Reilly DF, Brazis PW, Rubino FA (1982) The misdiagnosis of unilateral presentations of amyotrophic lateral sclerosis. Muscle Nerve 5:724-726
17. Orrell RW, Lane RJ, Guiloff RJ (1994) Recent developments in the drug treatment of motor neurone disease. BMJ 309: 140-141

18. Rowland LP (1995) Hereditary and acquired motor neuron diseases. In: Rowland LP (ed) Merrit's textbook of neurology. Ninth ed. Williams \& Wilkins, Baltimore, pp 743-749

19. Sanjak M, Paulson D, Sufit R, et al (1987) Physiologic and metabolic response to progressive and prolonged exercise in amyotrophic lateral sclerosis. Neurology 37: 1217-1220

20. Schiffer D, Chiò A, Giordana MT, et al (1991) Misdiagnosis in ALS: frequence and implications. Ital J Neurol Sci 12:25
21. Sufit R (1997) Symptomatic treatment of ALS. Neurology [suppl. 4] 48:S $15-\mathrm{S} 22$

22. Tyler HR, Shefner J (1991) Amyotrophic lateral sclerosis. In: De Jong JMBV (ed) Handbook of clinical neurology, vol 59; Diseases of the Motor System. North Holland Publ Co, Amsterdam, pp 169-215

23. Voltz R, Borasio GD (1997) Palliative therapy in the terminal stage of neurological diseases. J Neurol 244 [Suppl 4] : S 2-S 10 